This is an electronic reprint of the original article. This reprint may differ from the original in pagination and typographic detail.

Author(s): Lehrbäck, Juha

Title: Weighted Hardy inequalities beyond Lipschitz domains

Year: $\quad 2014$

Version:

Please cite the original version:

Lehrbäck, J. (2014). Weighted Hardy inequalities beyond Lipschitz domains.

Proceedings of the American Mathematical Society, 142(5), 1705-1715.

https://doi.org/10.1090/S0002-9939-2014-11904-6

All material supplied via JYX is protected by copyright and other intellectual property rights, and duplication or sale of all or part of any of the repository collections is not permitted, except that material may be duplicated by you for your research use or educational purposes in electronic or print form. You must obtain permission for any other use. Electronic or print copies may not be offered, whether for sale or otherwise to anyone who is not an authorised user. 


\title{
WEIGHTED HARDY INEQUALITIES BEYOND LIPSCHITZ DOMAINS
}

\author{
JUHA LEHRBÄCK
}

\begin{abstract}
It is a well-known fact that in a Lipschitz domain $\Omega \subset \mathbb{R}^{n}$ a $p$-Hardy inequality, with weight $\operatorname{dist}(x, \partial \Omega)^{\beta}$, holds for all $u \in C_{0}^{\infty}(\Omega)$ whenever $\beta<p-1$. We show that actually the same is true under the sole assumption that the boundary of the domain satisfies a uniform density condition with the exponent $\lambda=n-1$. Corresponding results also hold for smaller exponents, and, in fact, our methods work in general metric spaces satisfying standard structural assumptions.
\end{abstract}

\section{INTRODUCTION}

We say that an open set $\Omega \subset \mathbb{R}^{n}$ admits the $(p, \beta)$-Hardy inequality, for $1<p<\infty$ and $\beta \in \mathbb{R}$, if there exists a constant $C>0$ such that the inequality

$$
\int_{\Omega}|u(x)|^{p} d_{\Omega}(x)^{\beta-p} d x \leq C \int_{\Omega}|\nabla u(x)|^{p} d_{\Omega}(x)^{\beta} d x,
$$

where $d_{\Omega}(x)=\operatorname{dist}(x, \partial \Omega)$, holds for every $u \in C_{0}^{\infty}(\Omega)$. After the onedimensional considerations by G. H. Hardy et. al. in the early 20 th century (see $[6, \S 330]$ and the references therein), these inequlities were introduced in dimension $n \geq 2$ by J. Nečas. The main point of reflection for our studies and results is his theorem from [21] (see also A. Kufner [14] for this and related results):

Theorem 1.1 (Nečas 1962). Let $\Omega \subset \mathbb{R}^{n}$ be a bounded Lipschitz domain and let $1<p<\infty$. Then $\Omega$ admits the $(p, \beta)$-Hardy inequality for all $\beta<p-1$.

Recall that a domain (an open and connected set) is said to be a Lipschitz domain if the boundary $\partial \Omega$ can be represented locally as graphs of Lipschitz continuous functions. It follows from this definition that the boundary of a Lipschitz domain $\Omega \subset \mathbb{R}^{n}$ is both 'smooth' and 'thick', the latter for instance in the sense that

$$
\mathcal{H}_{\infty}^{n-1}\left(\partial \Omega \cap B\left(x, 2 d_{\Omega}(x)\right)\right) \geq C_{0} d_{\Omega}(x)^{n-1}
$$

for all $x \in \Omega$, where $\mathcal{H}_{\infty}^{\lambda}$ denotes the $\lambda$-dimensional Hausdorff content. Our main result is the following far-reaching generalization of Theorem 1.1.

Theorem 1.2. Let $\Omega \subset \mathbb{R}^{n}$ be an open set and let $1<p<\infty$. Suppose that there exist an exponent $0 \leq \lambda \leq n-1$ and a constant $C_{0}>0$ such that

$$
\mathcal{H}_{\infty}^{\lambda}\left(\partial \Omega \cap B\left(x, 2 d_{\Omega}(x)\right)\right) \geq C_{0} d_{\Omega}(x)^{\lambda}
$$

for all $x \in \Omega$. Then $\Omega$ admits the $(p, \beta)$-Hardy inequality for all $\beta<p-n+\lambda$.

2000 Mathematics Subject Classification. Primary 46E35, 26D15.

The author was supported in part by the Academy of Finland, grant no. 120972. 
We note that Theorem 1.2 was partly conjectured in [12]. By (2), Theorem 1.1 follows from Theorem 1.2 by taking $\lambda=n-1$. We conclude that the smoothness of a Lipschitz boundary plays no role here, as the thickness alone suffices for Hardy inequalities. Another important and interesting consequence of Theorem 1.2 is that each simply connected domain in $\mathbb{R}^{2}$ admits the $(p, \beta)$-Hardy (at least) for all $\beta<p-1$.

The bound $\beta<p-n+\lambda$ in Theorem 1.2 is optimal. In fact, it was shown in [17] (following the unweighted considerations from [13]) that if $\Omega$ has an isolated boundary part of (Hausdorff) dimension $\lambda$, then it is not possible for $\Omega$ to admit the $(p, p-n+\lambda)$-Hardy inequality, although the $(p, \beta)$-Hardy inequality might still hold for some larger $\beta$; see [17]. Also the bound $\lambda \leq n-1$ (whence $\beta<p-1$ ) is essential, as examples from [12] show.

Conditions of the type (3) are referred to as inner boundary density conditions'. By [15, Thm 1], such conditions are actually equivalent to similar density conditions for the complement $\Omega^{c}$. In particular, (3) holds for all $x \in \Omega$ with an exponent $\lambda>n-q$ if and only if $\Omega^{c}$ is uniformly $q$-fat (see e.g. [15] for the definition). It follows that we can rewrite Theorem 1.2 in the spirit of the unweighted results by A. Ancona [1] (the case $p=2$ ) and J. Lewis [19], and generalize the weighted inequalities of A. Wannebo [22], as follows:

Corollary 1.3. Let $\Omega \subset \mathbb{R}^{n}$ be an open set and assume that $\mathbb{R}^{n} \backslash \Omega$ is uniformly $q$-fat for all $q>s \geq 1$. Then $\Omega$ admits the $(p, \beta)$-Hardy inequality whenever $1<p<\infty$ and $\beta<p-s$.

To be precise, Wannebo proved in [22] that uniform $p$-fatness of the complement, for $1<p<\infty$, suffices for $(p, \beta)$-Hardy inequalities for all $\beta<\beta_{0}$, where $\beta_{0}$ is some small positive number. Hence, the main novelties in Corollary 1.3 are that we get an explicit and sharp bound for such an $\beta_{0}$ and that we can also deal with the cases when $p \leq s$, where $s$ is the 'optimal' fatness of the complement; of course, in such cases we must have $\beta<0$.

Our proof of Theorem 1.2 is based on rather standard 'geometric' ideas and methods, which actually work in the much more general setting of a metric measure space, provided that the space satisfies some structural conditions; see Section 2.1 for this general framework. In the case $\beta \leq 0$, Theorem 1.2 is an immediate consequence of a stronger (and for $\beta<0$ previously unknown) result concerning the so-called pointwise Hardy inequalities; see Section 2.4 for the definition and Theorem 4.2 for the result. In fact, Theorem 4.2 together with [18, Thm 3.1] shows that, for $\beta \leq 0$, condition (3) for some $\lambda>n-p+\beta$ is actually both necessary and sufficient for a domain $\Omega \subset \mathbb{R}^{n}$ to admit a pointwise version of the $(p, \beta)$-Hardy inequality (Corollary 4.4).

On the other hand, for $\beta>0$ density condition (3) with an exponent $n-p+\beta<\lambda \leq n$ is still necessary for pointwise inequalities by [18], but not anymore sufficient even for the usual Hardy inequality (1), as was shown by examples in [12]. Nevertheless, if $0<\beta<p-n+\lambda \leq p-1$ and (3) holds, then the $(p, \beta)$-Hardy inequality can be obtained from the unweighted $(p-\beta)$-Hardy inequality by an integration trick, and the theorem follows; see Section 4 for the details. 
In [12] it was actually shown that (3) together with an additional accessibility condition suffices for a pointwise $(p, \beta)$-Hardy inequality for all $\beta<p-n+\lambda$. By our Theorem 4.2, such an accessibility condition can now be dropped altogether if $\beta \leq 0$, and, by Theorem 1.2, for $0<\beta<p-1$ if we are only interested in the validity of the integral Hardy inequality (1). The main theorem of [12] concerning pointwise inequalities for $\beta>0$, with accessibility, is generalized to metric spaces in Theorem 4.5 with a simplified proof. As the examples from [12] did show that for $\beta \geq p-1$ density condition (3) alone is not sufficient for the pointwise $(p, \beta)$-Hardy inequality, we conclude that the only piece that is still missing from the complete picture is whether the assumptions of Theorem 1.2 always (that is, also for $0<\beta<p-n+\lambda \leq p-1)$ suffice for a pointwise version of the $(p, \beta)$-Hardy inequality. We conjecture that this is the case, and mention, for the record, that this question was really the essence of the (now proven) Conjecture 1.6 of [12].

The organization of this paper is as follows: We begin in Section 2 with basic definitions and other preliminaries on metric spaces, Hausdorff contents, and Hardy inequalities. Section 3 is devoted to the statement and proof of our main lemma, which is then used in Section 4 to prove the results on Hardy inequalities. For notation we remark that throughout the paper the letter $C$ is used to denote positive constants whose value may change from expression to expression.

\section{Preliminaries}

2.1. Metric spaces. We assume that $X=(X, d, \mu)$ is a complete metric measure space equipped with a metric $d$ and a Borel regular outer measure $\mu$ such that $0<\mu(B)<\infty$ for all balls $B=B(x, r)=\{y \in X: d(x, y)<r\}$. For $0<t<\infty$, we write $t B=B(x, t r)$, and $\bar{B}$ is the corresponding closed ball. When $A \subset X, \partial A$ is the boundary and $\bar{A}$ the closure of $A$. The distance from $x \in X$ to $A \subset X$ is denoted $d(x, A)$. When $\Omega \subset X$ is an open set and $x \in \Omega$, we also denote $d_{\Omega}(x)=d(x, \partial \Omega)$.

We assume that the measure $\mu$ is doubling, i.e. that there exists a constant $C_{d} \geq 1$ such that

$$
\mu(2 B) \leq C_{d} \mu(B)
$$

for all balls $B$ of $X$. The doubling condition together with the completeness implies that the space $X$ is proper, that is, closed balls of $X$ are compact.

The doubling condition gives an upper bound for the dimension of $X$ in the sense that there is a constant $C=C\left(C_{d}\right)>0$ such that, for $s=\log _{2} C_{d}$,

$$
\frac{\mu(B(y, r))}{\mu(B(x, R))} \geq C\left(\frac{r}{R}\right)^{s}
$$

whenever $0<r \leq R<\operatorname{diam} X$ and $y \in B(x, R)$. The infimum of the exponents $s$ for which (4) holds is called the doubling dimension of $X$.

Another crucial assumption is that the space $X$ supports a (weak) $(1, p)$ Poincaré inequality. More precisely, we assume that there exist constants $C>0$ and $\tau \geq 1$ such that for all balls $B \subset X$, all continuous functions $u$, 
and for all upper gradients $g_{u}$ of $u$, we have the inequality

$$
f_{B}\left|u-u_{B}\right| d \mu \leq C r\left(f_{\tau B} g_{u}^{p} d \mu\right)^{1 / p}
$$

where

$$
u_{B}=f_{B} u d \mu=\mu(B)^{-1} \int_{B} u d \mu
$$

is the integral average of $u$ over $B$. Recall that a Borel function $g \geq 0$ is said to be an upper gradient of a function $u$ (on an open set $\Omega \subset X$ ), if for all curves $\gamma$ joining points $x$ and $y$ (in $\Omega$ ) we have

$$
|u(x)-u(y)| \leq \int_{\gamma} g d s
$$

whenever both $u(x)$ and $u(y)$ are finite, and $\int_{\gamma} g d s=\infty$ otherwise. By a curve we simply mean a nonconstant, rectifiable, continuous mapping from a compact interval to $X$.

Examples of metric spaces satisfying the above conditions include (weighted) Euclidean spaces, compact Riemannian manifolds, Carnot groups, and metric graphs. See for instance [3], [5], [7], and the references therein for more information on analysis on metric spaces based on upper gradients and Poincaré inequalities.

For the rest of the paper we explicitly assume that in the context of the $(p, \beta)$-Hardy inequality the space $X$ supports a $(1, p)$-Poincaré inequality. However, in our proofs we sometimes need to use a $(1, q)$-Poincaré inequality for an exponent $q<p$, but this is justified by the self-improvement property of Poincaré inequalities, see [9].

Recall that a function $u: \Omega \rightarrow \mathbb{R}$ is said to be $(L-)$ Lipschitz, if

$$
|u(x)-u(y)| \leq L d(x, y) \quad \text { for all } x, y \in \Omega .
$$

The set of all Lipschitz functions $u: \Omega \rightarrow \mathbb{R}$ is denoted $\operatorname{Lip}(\Omega)$, and $\operatorname{Lip}_{0}(\Omega)$ is the set of Lipschitz functions $u \in \operatorname{Lip}(\Omega)$ with a compact support in $\Omega$. Recall that the support of a function $u: \Omega \rightarrow \mathbb{R}$ is the closure of the set where $u$ is non-zero. It is straight-forward to check that the pointwise Lipschitz constant

$$
\operatorname{Lip}(u ; x)=\limsup _{y \rightarrow x} \frac{|u(x)-u(y)|}{d(x, y)}
$$

defines an upper gradient $g$ for a Lipschitz function $u: \Omega \rightarrow \mathbb{R}$ by $g(x)=$ $\operatorname{Lip}(u ; x)$.

2.2. Hausdorff contents. We measure the thickness of sets $E \subset X$ by means of Hausdorff contents. The usual $\lambda$-Hausdorff content of a set $A \subset X$ is defined by

$$
\mathcal{H}_{\infty}^{\lambda}(A)=\inf \left\{\sum_{k=1}^{\infty} r_{k}^{\lambda}: A \subset \bigcup_{k=1}^{\infty} B\left(x_{k}, r_{k}\right), x_{k} \in A\right\},
$$

and the Hausdorff dimension of $A$ is then

$$
\operatorname{dim}(A)=\inf \left\{\lambda>0: \mathcal{H}_{\infty}^{\lambda}(A)=0\right\} .
$$


However, in general metric spaces it is often more convenient to use a modified version of $\mathcal{H}_{\infty}^{\lambda}$, namely the Hausdorff content of codimension $t$, which is given for a set $E \subset X$ by

$$
\mathscr{H}_{R}^{t}(E)=\inf \left\{\sum_{i \in I} \mu\left(B\left(x_{i}, r_{i}\right)\right) r_{i}^{-t}: E \subset \bigcup_{i \in I} B\left(x_{i}, r_{i}\right), r_{i} \leq R\right\} .
$$

Here we may again assume that $x_{i} \in E$, as this increases $\mathscr{H}_{R}^{t}(E)$ at most by a constant factor.

A metric space $X$ is said to be (Ahlfors) $Q$-reqular if there are constants $c_{1}, c_{2}>0$ such that

$$
c_{1} r^{Q} \leq \mu(B(x, r)) \leq c_{2} r^{Q}
$$

for all balls $B(x, r)$ in $X$. It is easy to see that in a $Q$-regular space $X$ the content $\mathscr{H}_{\infty}^{t}(E)$ is comparable with the usual Hausdorff content $\mathcal{H}_{\infty}^{Q-t}(E)$ for every $E \subset X$.

2.3. Chain condition. We introduce an important chain condition following [4]; see also [7]. Let $x \in \Omega \subset X$, where $\Omega$ is an open set, and take $\lambda, M \geq 1$ and $a>1$. We say that $w \in \bar{\Omega}$ is connected to $x$ by a $(\lambda, M, a)$ chain in $\Omega$, denoted $w \in \mathcal{C}_{\Omega}(\lambda, M, a ; x)$, if there exists a sequence of balls $B_{k}=B\left(x_{k}, r_{k}\right), k=0,1,2, \ldots$, so that $x_{0}=x$ and $x_{k} \rightarrow w$ as $k \rightarrow \infty$, and the following conditions hold for each $k=0,1,2, \ldots$ :

(i) $\lambda B_{k} \subset \Omega$;

(ii) $M^{-1} d_{\Omega}(x) a^{-k} \leq r_{k} \leq M d_{\Omega}(x) a^{-k}$;

(iii) there is a ball $B_{k}^{\prime}$ so that $B_{k}^{\prime} \subset B_{k} \cap B_{k+1} \subset M B_{k}^{\prime}$.

For instance, if $\Omega \subset X$ is a $C_{J^{-}}$John domain with center point $x$, and $\lambda \geq 1$, then there exists $M \geq 1$, depending on $\lambda, C_{J}$, and the doubling constant such that $w \in \mathcal{C}_{\Omega}(\lambda, M, 2 ; x)$ for each $w \in \bar{\Omega}$ (see [5, Thm 9.3]). We mention that in $\mathbb{R}^{n}$ the sets $\mathcal{C}_{\Omega}(\lambda, M, 2 ; x) \cap \partial \Omega$ agree with suitable $c$-visual boundaries near $x$, defined in [12], where $c, \lambda$, and $M$ only depend on each other and $n$, and we may actually choose $\lambda$ to be as large as we want.

2.4. Hardy inequalities. In the setting of a general metric space the $(p, \beta)$ Hardy inequality takes the following form:

$$
\int_{\Omega}|u|^{p} d_{\Omega}^{\beta-p} d \mu \leq C \int_{\Omega} g_{u}^{p} d_{\Omega}^{\beta} d \mu
$$

where $g_{u}$ is an upper gradient of $u$. We say that an open set $\Omega \subset X$ admits the (metric) $(p, \beta)$-Hardy inequality if there exists a constant $C>0$ so that (7) holds for every $u \in \operatorname{Lip}_{0}(\Omega)$ and for all upper gradients $g_{u}$ of $u$.

Following the unweighted considerations by Hajłasz [2] and Kinnunen and Martio [10], a pointwise version of the weighted $(p, \beta)$-Hardy inequality (1) was introduced in [12]. The metric space version reads as follows:

$$
|u(x)| \leq C d_{\Omega}(x)^{1-\frac{\beta}{p}}\left(M_{L d_{\Omega}(x)}\left(g_{u}^{q} d_{\Omega}^{\frac{\beta}{p} q}\right)(x)\right)^{1 / q},
$$

where $1<q<p, L \geq 1$, and $M_{R}$ is the usual restricted Hardy-Littlewood maximal operator, defined by

$$
M_{R} f(x)=\sup _{0<r \leq R} f_{B(x, r)}|f(y)| d \mu
$$


for $f \in L_{\text {loc }}^{1}(X)$. We say that an open set $\Omega \subset X$ admits the pointwise $(p, \beta)$-Hardy inequality if there exist some $1<q<p$ and constants $C>0$, $L \geq 1$ so that the inequality (8) holds for all $u \in \operatorname{Lip}_{0}(\Omega)$ with these $q, C$, and $L$.

Remark. Using the maximal theorem, it is easy to see that if the pointwise inequality (8) holds for a function $u$ at (almost) every $x \in \Omega$, then the usual $(p, \beta)$-Hardy inequality holds for $u$ with a constant only depending on $p$ and the constants from the pointwise inequality and the maximal function inequality (cf. [12]).

\section{Main Lemma}

The proofs of our main results are based on the following local estimates for Lipschitz functions vanishing at the boundary. For weight exponents $\beta \leq 0$ the estimate involves the whole boundary near a point $x \in \Omega$, whereas for $\beta>0$ we need to restrict to the part of the boundary that we can connect to $x \in \Omega$ with good chains of balls (cf. Section 2.3).

Lemma 3.1. Let $1<p<\infty$ and $\beta<p$, let $\Omega \subset X$ be an open set, and take $x \in \Omega$. Denote $B(x)=\bar{B}\left(x, d_{\Omega}(x)\right)$, and define

(a) $E=\partial \Omega \cap 2 B(x)$ if $\beta \leq 0$,

(b) $E=\mathcal{C}_{\Omega}(\lambda, M, a ; x) \cap \partial \Omega$ if $0<\beta<p$, where $\lambda \geq 2 \tau$ ( $\tau$ is from inequality (5)).

Then, for each $0 \leq t<p-\beta$, there exist an exponent $1<q<p$ and constants $C>0, L \geq 1$, all independent of $x$, such that the estimate

$$
\mathscr{H}_{d_{\Omega}(x)}^{t}(E)\left|u_{(2 \tau)^{-1} B(x)}\right|^{q} \leq C d_{\Omega}(x)^{q-\beta \frac{q}{p}-t} \int_{L B(x)} g_{u}(y)^{q} d_{\Omega}(y)^{\beta \frac{q}{p}} d \mu
$$

holds for every $u \in \operatorname{Lip}_{0}(\Omega)$.

Proof. Our proof combines elements from the proofs of [12, Lemma 5.2] and [8, Thm 5.9]. Let $0 \leq t<p-\beta$. It is easy to check that we can choose $1<q<\infty$ so that

$$
\frac{p}{p-\beta} t<q<p .
$$

Moreover, we may assume that $X$ supports a $(1, q)$-Poincaré inequality (cf. [9]). Also denote $\beta^{\prime}=\frac{q}{p} \beta$. Then $q / p>t /(p-\beta)$, and we have

$$
q-\beta^{\prime}-t=\frac{q}{p}(p-\beta)-t>0 .
$$

Denote $R=d_{\Omega}(x)$ and $B=\bar{B}(x, R)$. If $u_{(2 \tau)^{-1} B}=0$ the claim (9) is trivial, so we may assume that $\left|u_{(2 \tau)^{-1} B}\right|>0$, and in fact, by homogeneity, that $\left|u_{(2 \tau)^{-1} B}\right|=1$. It is also clear that we may assume $E \neq \emptyset$.

Part (b): Let us start with the more complicated part (b). The proof of part (a) goes along the same lines; we comment on the differences at the end of the proof.

First notice that, by the properties of the chains (Section 2.3), there exists $L_{0} \geq 1$, independent of $x$, such that $\mathcal{C}_{\Omega}(\lambda, M, a ; x) \subset L_{0} B$. Now fix 
$w \in \mathcal{C}_{\Omega}(\lambda, M, a ; x) \cap \partial \Omega$ and let $B_{i}=B\left(x_{i}, r_{i}\right)$ be the corresponding chain of balls. Then

$$
1 \leq\left|u(w)-u_{(2 \tau)^{-1} B}\right| \leq\left|u_{B_{0}}\right|+\left|u_{B_{0}}-u_{(2 \tau)^{-1} B}\right|,
$$

and it follows from the properties of the chain and the assumption $2 \tau \leq \lambda$ that $B_{0} \subset(2 \tau)^{-1} B$. If $\left|u_{B_{0}}\right|<1 / 2$, we infer, using the above facts and the $(1, q)$-Poincaré inequality, that

$$
\begin{aligned}
\frac{1}{2} & \leq\left|u_{B_{0}}-u_{(2 \tau)^{-1} B}\right| \leq C R\left(f_{(1 / 2) B} g_{u}(y)^{q} d \mu\right)^{1 / q} \\
& \leq C R^{1-\beta / p}\left(f_{(1 / 2) B} g_{u}(y)^{q} d_{\Omega}(y)^{\beta \frac{q}{p}} d \mu\right)^{1 / q} .
\end{aligned}
$$

As $\mathscr{H}_{R}^{t}(E) \leq C \mu(B) R^{-t}$, and $\left|u_{(2 \tau)^{-1} B}\right|=1$, the claim (9) easily follows from the doubling condition.

We may hence assume that $1 / 2 \leq\left|u_{B_{0}}\right|=\left|u(w)-u_{B_{0}}\right|$ for every $w \in$ $\mathcal{C}_{\Omega}(\lambda, M, a ; x) \cap \partial \Omega$. Using the properties of the chain together with the $(1, q)$-Poincaré inequality and the doubling condition, and the assumption that the support of $u$ is compact, we get the standard estimate (see for example [4])

$$
1 \leq C \sum_{k=0}^{\infty} r_{k}\left(f_{\tau B_{k}} g_{u}^{q} d \mu\right)^{1 / q} .
$$

From (12) it follows that there must be a constant $C_{1}>0$, independent of $x, u$, and $w$, and at least one index $k_{w} \in \mathbb{N}$ so that

$$
r_{k_{w}}\left(f_{\tau B_{k_{w}}} g_{u}^{q} d \mu\right)^{1 / q} \geq C_{1} a^{-k_{w} \alpha}=C_{1} R^{-\alpha}{r_{k_{w}}}{ }^{\alpha},
$$

where we choose $\alpha=\frac{1}{q}\left(q-\beta^{\prime}-t\right)>0$ (by (10)). Let us write from now on $B_{w}=B\left(x_{w}, r_{w}\right)$ instead of $B_{k_{w}}=B\left(x_{k_{w}}, r_{k_{w}}\right)$.

We assumed that $\tau B_{w} \subset(\lambda / 2) B_{w}$, and as $\lambda B_{w} \subset \Omega$, it follows that $r_{w}{ }^{\beta} \leq C d_{\Omega}(y)^{\beta}$ for each $y \in \tau B_{w}$. Thus

$$
\left(f_{\tau B_{w}} g_{u}^{q} d \mu\right)^{1 / q} \leq C r_{w}^{-\beta / p} \mu\left(\tau B_{w}\right)^{-1 / q}\left(\int_{\tau B_{w}} g_{u}(y)^{q} d_{\Omega}(y)^{\beta \frac{q}{p}} d \mu\right)^{1 / q}
$$

In particular, combining (13) and (14) we obtain for each $w \in E$ a ball $B_{w}$ such that

$$
\mu\left(\tau B_{w}\right)^{1 / q} r_{w}^{\alpha-1+\beta / p} \leq C R^{\alpha}\left(\int_{\tau B_{w}} g_{u}(y)^{q} d_{\Omega}(y)^{\beta^{\prime}} d \mu\right)^{1 / q} .
$$

But here $\alpha-1+\beta / p=t / q$, so by raising both sides of (15) to power $q$ we get a useful estimate

$$
\mu\left(\tau B_{w}\right) r_{w}^{-t} \leq C R^{q-\beta^{\prime}-t} \int_{\tau B_{w}} g_{u}(y)^{q} d_{\Omega}(y)^{\beta^{\prime}} d \mu .
$$

Using again the properties of the chain we see that there exists $\tau^{\prime} \geq \tau$ such that $\tau B_{w} \subset B\left(w, \tau^{\prime} r_{w}\right)$ holds for all $w \in E$. By the standard $5 r$ covering lemma (see e.g. [7]), there exist points $w_{1}, w_{2}, \ldots \in E$ so that if we denote $r_{i}=\tau^{\prime} r_{w_{i}}$, then the balls $\tilde{B}_{i}=B\left(w_{i}, r_{i}\right)$ are pairwise disjoint, but 
still $E \subset \bigcup_{i=1}^{\infty} 5 \tilde{B}_{i}$. Moreover, it is easy to find $L \geq 1$, independent of $x$, so that $\tilde{B}_{i} \subset L B$ for all $i$; recall that $B=\bar{B}\left(x, d_{\Omega}(x)\right)$. Estimate (16), the doubling property, and the pairwise disjointness of the balls $\tau B_{w_{i}} \subset \tilde{B}_{i} \subset$ $L B$ immediately yield

$$
\begin{aligned}
\mathscr{H}_{R}^{t}(E) & \leq \sum_{i=1}^{\infty} \mu\left(5 \tilde{B}_{i}\right)\left(5 r_{i}\right)^{-t} \leq C \sum_{i=1}^{\infty} \mu\left(\tau B_{w_{i}}\right) r_{w_{i}}^{-t} \\
& \leq \sum_{i=1}^{\infty} C R^{q-\beta^{\prime}-t} \int_{\tau B_{w_{i}}} g_{u}(y)^{q} d \Omega(y)^{\beta^{\prime}} d \mu \\
& \leq C R^{q-\beta^{\prime}-t} \int_{L B} g_{u}(y)^{q} d_{\Omega}(y)^{\beta^{\prime}} d \mu .
\end{aligned}
$$

As we assumed $\left|u_{(2 \tau)^{-1} B}\right|=1$ and denoted $\beta^{\prime}=\beta \frac{q}{p}$, estimate (9) for part (b) is proven.

Part (a): Let us only give here a brief description of the main differences compared to part (b). We begin by fixing $w \in E=\partial \Omega \cap 2 B$, then define $r_{k}=2^{-k} R, k \in \mathbb{N}$, and denote $B_{k}=B\left(w, r_{k}\right)$.

If $\left|u_{B_{0}}\right|<1 / 2$, we see with a calculation similar to (11) and using the inclusion $B_{0} \subset 3 B$ that

$$
1 \leq C R^{1-\beta / p}\left(f_{3 \tau B} g_{u}(y)^{q} d_{\Omega}(y)^{\beta \frac{q}{p}} d \mu\right)^{1 / q} .
$$

Notice that the assumption $\beta \leq 0$ guarantees that $R^{\beta} \leq C d_{\Omega}(y)^{\beta}$ for each $y \in 3 \tau B$. The claim (9) follows.

We may hence assume that $1 / 2 \leq\left|u_{B_{0}}\right|=\left|u(w)-u_{B_{0}}\right|$. But now estimate (12) follows again for balls $B_{k}$ by a standard 'telescoping' argument using the $(1, q)$-Poincaré inequality (cf. e.g. [7]), and the rest of the proof is almost identical to part (b); for instance, (13) holds now with $a=2$. Notice in particular that since $\beta \leq 0$ and $w \in E$, it follows again that $r_{k}{ }^{\beta} \leq d_{\Omega}(y)^{\beta}$ for each $y \in B_{k}$. At the end we can use the $5 r$-covering theorem directly to balls $\tau B_{w}$, as they are now centered at $w$, and the desired Hausdorff content estimate follows just as in (17). The proof is complete.

\section{Weighted AND POINTWISE INEQUALITIES}

Let us begin this section by rephrasing Theorem 1.2 in general metric spaces:

Theorem 4.1. Let $\Omega \subset X$ be an open set and let $1<p<\infty$. Suppose that there exist an exponent $t \geq 1$ and a constant $C_{0}>0$ such that

$$
\mathscr{H}_{d_{\Omega}(x)}^{t}\left(\partial \Omega \cap \bar{B}\left(x, 2 d_{\Omega}(x)\right)\right) \geq C_{0} \mu\left(\bar{B}\left(x, d_{\Omega}(x)\right)\right) d_{\Omega}(x)^{-t}
$$

for all $x \in \Omega$. Then $\Omega$ admits the $(p, \beta)$-Hardy inequality for all $\beta<p-t$.

In the case $\beta \leq 0$, Theorem 4.1 is an immediate consequence of the following result on pointwise inequalities. The special case $\beta=0$ of Theorem 4.2 is contained in the results of [11].

Theorem 4.2. Let $\Omega \subset X$ be an open set and let $1<p<\infty$ and $\beta \leq 0$. Suppose that there exist an exponent $0 \leq t<p-\beta$ and a constant $C_{0}>0$ 
such that the density condition (18) holds for all $x \in \Omega$. Then $\Omega$ admits the pointwise $(p, \beta)$-Hardy inequality.

Proof. Let $u \in \operatorname{Lip}_{0}(\Omega), x \in \Omega$, and denote $R=d_{\Omega}(x), B=B\left(x,(2 \tau)^{-1} R\right)$. Then

$$
|u(x)| \leq\left|u(x)-u_{B}\right|+\left|u_{B}\right| .
$$

Choose $1<q<p$ just as in the proof of Lemma 3.1. Again a standard telescoping trick using the $(1, q)$-Poincaré inequality gives

$$
\begin{aligned}
\left|u(x)-u_{B}\right| & \leq C R\left(M_{R / 2} g_{u}^{q}(x)\right)^{1 / q} \\
& \leq C R^{1-\beta / p}\left(M_{R / 2}\left(g_{u}^{q} d_{\Omega}^{\beta q / p}\right)(x)\right)^{1 / q} .
\end{aligned}
$$

On the other hand, using assumption (18), part (a) of Lemma 3.1, and the doubling condition, we obtain

$$
\begin{aligned}
\left|u_{B}\right|^{q} & \leq C \mu(\bar{B}(x, R))^{-1} R^{t} R^{q-\beta \frac{q}{p}-t} \int_{B(x, L R)} g_{u}(y)^{q} d_{\Omega}(y)^{\beta \frac{q}{p}} d \mu \\
& \leq C R^{q-\beta \frac{q}{p}} M_{L R}\left(g_{u}^{q} d_{\Omega}{ }^{\beta \frac{q}{p}}\right)(x) .
\end{aligned}
$$

The pointwise $(p, \beta)$-Hardy inequality follows easily from the previous estimates.

Conversely, we have the following necessary condition for pointwise Hardy inequalities. The Euclidean case was proven in [18], and the special case $\beta=0$ was done in [11] in the metric space setting. We omit the proof here, as the modifications needed to the proofs in [18] and [11] are obvious.

Proposition 4.3. Let $\Omega \subset X$ be an open set and assume that $\Omega$ admits the pointwise $(p, \beta)$-Hardy inequality (8). If $\beta<0$, we assume in addition that $X$ is $Q$-regular. Then there exists $t<p-\beta$ such that the inner density condition (18) holds for all $x \in \Omega$.

Combining Theorem 4.2 and Proposition 4.3 we obtain a characterization in the case $\beta<0$, which is new even in $\mathbb{R}^{n}$; for $\beta=0$ the corresponding result holds in metric spaces by [11].

Corollary 4.4. Assume that $X$ is $Q$-regular. Let $\Omega \subset X$ be an open set and let $1<p<\infty$ and $\beta<0$. Then $\Omega$ admits the pointwise $(p, \beta)$-Hardy inequality (8) if and only if there exist an exponent $t<p-\beta$ and a constant $C_{0}>0$ such that the inner density condition (18) holds for all $x \in \Omega$.

Let us finally give a proof for our main result concerning weighted Hardy inequalities:

Proof of Theorem 4.1. As the pointwise $(p, \beta)$-Hardy inequality always implies the usual $(p, \beta)$-Hardy inequality (see the remark at the end of Section 2.4), Theorem 4.1 follows from Theorem 4.2 for $\beta \leq 0$.

Hence, we only need to consider the case $0<\beta<p-t$. But now $p-\beta>t \geq 1$, and so Theorem 4.2, applied to the unweighted case, implies that $\Omega$ admits the $(p-\beta, 0)$-Hardy inequality. The claim now follows in fact from a straight-forward metric space generalization of a more general result of [16, Lemma 2.1], but let us recall here the calculations in this special case 
for the sake of completeness and in order to emphasize the role of elementary tools behind the theorem.

To this end, let $u \in \operatorname{Lip}_{0}(\Omega)$ and let $g_{u}$ be an upper gradient of $u$. Now define $v=|u|^{\frac{p}{p-\beta}}$. As $\beta>0$, we see that $v$ is a Lipschitz-function with a compact support in $\Omega$, and, moreover,

$$
g_{v}(x)=\left(\frac{p}{p-\beta}\right)|u(x)|^{\beta /(p-\beta)} g_{u}(x)
$$

defines an upper gradient for $v$. As the $(p-\beta, 0)$-Hardy inequality holds for $v$, we obtain, with the help of Hölder's inequality (observe $\frac{p-\beta}{p}+\frac{\beta}{p}=1$ ), that

$$
\begin{aligned}
\int_{\Omega}|u(x)|^{p} & d_{\Omega}(x)^{-(p-\beta)} d \mu=\int_{\Omega}|v(x)|^{p-\beta} d_{\Omega}(x)^{-(p-\beta)} d \mu \\
\leq & C_{1} \int_{\Omega} g_{v}(x)^{p-\beta} d \mu=C_{2} \int_{\Omega}|u(x)|^{\beta} g_{u}(x)^{p-\beta} d \mu \\
& =C_{2} \int_{\Omega}\left(|u(x)|^{\beta} d_{\Omega}(x)^{\frac{\beta(\beta-p)}{p}}\right)\left(g_{u}(x)^{p-\beta} d_{\Omega}(x)^{\frac{\beta(p-\beta)}{p}}\right) d \mu \\
& \leq C_{2}\left(\int_{\Omega}|u(x)|^{p} d_{\Omega}(x)^{\beta-p} d \mu\right)^{\frac{\beta}{p}}\left(\int_{\Omega} g_{u}(x)^{p} d_{\Omega}(x)^{\beta} d \mu\right)^{\frac{p-\beta}{p}} .
\end{aligned}
$$

From (19) the $(p, \beta)$-Hardy inequality for $u$ easily follows by first dividing with the first integral term on the right-hand side (which we may assume to be non-zero), and then taking both sides to power $p /(p-\beta)$.

Remark. There is an interesting observation concerning the procedure in (19) and the best possible constants in Hardy inequalities. Namely, it is wellknown that the best possible constant for the $p$-Hardy inequality in a convex domain $\Omega \subset \mathbb{R}^{n}, n \geq 1$, is $C_{1}=(p /(p-1))^{p}$, and in other smooth domains the constant is in general larger; see e.g. [6] $(n=1)$ and [20] $(n \geq 2)$. On the other hand, the best possible constant for the one-dimensional weighted $(p, \beta)$-Hardy inequality, with $\beta<p-1$, is $(p /(p-\beta-1))^{p}$ (see [6]), and this can be trivially generalized to, say, a ball or a half-space in $\mathbb{R}^{n}$.

Usually, our methods on Hardy inequalities lead to constants which are far from being optimal. However, if we have in (19) that $C_{1}=\left(\frac{p-\beta}{p-\beta-1}\right)^{p-\beta}$ (the optimal constant for the $(p-\beta)$-Hardy), we see directly from the calculation that the constant in the corresponding $(p, \beta)$-Hardy inequality will then be $C=\left(\frac{p}{p-\beta-1}\right)^{p}$, which is, at least in the above special cases, the best possible constant for the $(p, \beta)$-Hardy inequality. This raises the question whether the above procedure preserves the optimal constants in (weighted) Hardy inequalities even in more general cases.

We finish by recording the following result, which coincides in the case $X=\mathbb{R}^{n}$ with the main theorem of [12]. The proof is identical to the proof of Theorem 4.2 besides that the part (b) of Lemma 3.1 (instead of part (a)) is needed. When $X=\mathbb{R}^{n}$, the derivation of this result using Lemma 3.1(b) is - once all the technicalities arising from the metric space setting are removed - in a theoretical sense a simplification over the proof from [12], as here we avoid completely the use of Whitney type coverings and Frostman's lemma. 
Theorem 4.5. Let $\Omega \subset X$ be an open set and let $1<p<\infty$. Suppose that there exist an exponent $t \geq 0$ and constants $C_{0}>0, M \geq 1, \lambda \geq 2 \tau$, and $a>1$ such that

$$
\mathscr{H}_{d_{\Omega}(x)}^{t}\left(\mathcal{C}_{\Omega}(\lambda, M, a ; x) \cap \partial \Omega\right) \geq C_{0} \mu\left(\bar{B}\left(x, d_{\Omega}(x)\right)\right) d_{\Omega}(x)^{-t}
$$

for all $x \in \Omega$. Then $\Omega$ admits the pointwise $(p, \beta)$-Hardy inequality for all $\beta<p-t$, and hence also the usual $(p, \beta)$-Hardy inequality for these $\beta$.

Notice that, contrary to Theorem 4.1, we do not need to assume above that $t \geq 1$, only $t \geq 0$. Moreover, as noted in the Introduction, examples from [12] show that some kind of an 'accessibility' condition is needed to guarantee even the validity of the usual integral Hardy inequalities when $\beta \geq p-1$ (corresponding to the case $0 \leq t<1$ ). As a concrete example we mention that if $\Omega \subset \mathbb{R}^{n}$ is a uniform domain satisfying (18) for all $x \in \Omega$, then also (20) holds with suitable constants for all $x \in \Omega$; see [12] for details. In particular, this is true in a snowflake domain, where we can have $t<1$.

Acknowledgement. The author is grateful to Professor Pekka Koskela for helpful comments during the preparation of this work.

\section{REFERENCES}

[1] A. Ancona, 'On strong barriers and an inequality of Hardy for domains in $\mathbb{R}^{n}, J$. London Math. Soc. (2) 34 (1986), no. 2, 274-290.

[2] P. HajŁasz, 'Pointwise Hardy inequalities', Proc. Amer. Math. Soc. 127 (1999), no. $2,417-423$.

[3] P. HajŁasz, 'Sobolev spaces on metric-measure spaces', In: "Heat kernels and analysis on manifolds, graphs, and metric spaces", (Paris, 2002), 173-218, Contemp. Math. 338, Amer. Math. Soc. Providence, RI, 2003.

[4] P. Hajeasz and P. Koskela, 'Sobolev meets Poincaré', C. R. Acad. Sci. Paris Sér. A Math. 320 (1995), 1211-1215.

[5] P. Hajeasz and P. Koskela, 'Sobolev met Poincaré', Mem. Amer. Math. Soc. 145 (2000), no. 688.

[6] G. H. Hardy, J. E. Littlewood and G. Pólya, 'Inequalities', (Second edition), Cambridge University Press, Cambridge, 1952.

[7] J. Heinonen, 'Lectures on analysis on metric spaces', Universitext, Springer-Verlag, New York, 2001.

[8] J. Heinonen and P. Koskela, 'Quasiconformal maps in metric spaces with controlled geometry', Acta Math. 181 (1998), no. 1, 1-61.

[9] S. Keith And X. Zhong, 'The Poincaré inequality is an open ended condition', Ann. of Math. (2) 167 (2008), no.2, 575-599.

[10] J. Kinnunen and O. Martio, 'Hardy's inequalities for Sobolev functions', Math. Res. Lett. 4 (1997), no. 4, 489-500.

[11] R. Korte, J. Lehrbäck, And H. TuOminen, 'The equivalence between pointwise Hardy inequalities and uniform fatness', Math. Ann. 351 (2011), no. 3, 711-731.

[12] P. Koskela And J. Lehrbäck, 'Weighted pointwise Hardy inequalities', J. London Math. Soc. (2) 79 (2009), no. 3, 757-779.

[13] P. Koskela and X. Zhong, 'Hardy's inequality and the boundary size', Proc. Amer. Math. Soc. 131 (2003), no. 4, 1151-1158.

[14] A. Kufner, 'Weighted Sobolev spaces', Wiley, New York, 1985.

[15] J. Lehrbäck, 'Pointwise Hardy inequalities and uniformly fat sets', Proc. Amer. Math. Soc. 136 (2008), no. 6, 2193-2200.

[16] J. LehrвÄCK, 'Self-improving properties of weighted Hardy inequalities', Adv. Calc. Var. 1 (2008), no. 2, 193-203.

[17] J. Lenrbäck, 'Weighted Hardy inequalities and the size of the boundary', Manuscripta Math. 127 (2008), no. 2, 249-273. 
[18] J. LEHRB ̈̈CK, 'Necessary conditions for weighted pointwise Hardy inequalities', Ann. Acad. Sci. Fenn. Math. 34 (2009), no. 2, 437-446.

[19] J. L. Lewis, 'Uniformly fat sets', Trans. Amer. Math. Soc. 308 (1988), no. 1, 177-196.

[20] M. Marcus, V. J. Mizel and Y. Pinchover, 'On the best constant for Hardy's inequality in $\mathbb{R}^{n}$, Trans. Amer. Math. Soc. 350 (1998), no. 8, 3237-3255.

[21] J. NEČAS, 'Sur une méthode pour résoudre les équations aux dérivées partielles du type elliptique, voisine de la variationnelle', Ann. Scuola Norm. Sup. Pisa (3) 16 (1962), 305-326.

[22] A. Wannebo, 'Hardy inequalities', Proc. Amer. Math. Soc. 109 (1990), 85-95.

Department of Mathematics and Statistics, P.O. Box 35 (MaD), FiN-40014 UNIVERSITY OF JYVÄSKYLÄ, FinLAND

E-mail address: juha.lehrback@jyu.fi 